\title{
Synopsis of Acanthocerini (Hemiptera, Coreidae) from Argentina
}

\author{
José Luis Pall ${ }^{1,2}$, María del Carmen Coscarón' \\ I División Entomología, Facultad de Ciencias Naturales y Museo, Universidad Nacional de La Palta, Paseo del \\ Bosque, CP 1900, La Plata, Argentina 2 Facultad de Ciencias Exactas y Naturales, Universidad Nacional de \\ La Pampa, Argentina, Uruguay 151 L6300CLB, Santa Rosa, La Pampa, Argentina
}

Corresponding author: José Luis Pall (mcoscaron@fcnym.unlp.edu.ar; pall.joseluis@gmail.com)

Academic editor: Thomas Henry | Received 25 July 2012 | Accepted 7 May 2013 | Published 29 May 2013

Citation: José Luis Pall, María del Camen Coscarón M (2013) Synopsis of Acanthocerini (Hemiptera, Coreidae) from Argentina. ZooKeys 305: 33-53. doi: 10.3897/zookeys.305.3727

\begin{abstract}
Eight genera and 13 species of the tribe Acanthocerini are recorded in Argentina, i.e., Athaumastus haematicus (Stål), A. macer Brailovsky, A. subcarinatus (Stål), A. subterlineatus Bergroth, Beutelspacoris sanchezi Brailovsky, B. dilatata Casini, Camptischium clavipes (Fabricius), Crinocerus sanctus (Fabricius), Dersagrena flaviventris (Berg), D. lacerdae (Signoret), D. subfoveolata (Berg), Thlastocoris hernandezi Brailovsky and Zoreva dentipes Fabricius. Redescriptions are given for A. haematicus, A. subcarinatus and D. flaviventris with photographs of male and female genitalia of D. subfoveolata. Zoreva recorded from Argentina the first time. New locality records are given for Buenos Aires, Chaco, Formosa, Misiones, and Tucumán.
\end{abstract}

\section{Keywords}

Acanthocerini, genera, Argentina, key, redescription, distribution 


\section{Introduction}

The Coreidae, commonly called "leaf-footed, pod- or squash-bugs", are heavy bodied insects usually strongly elongate or broadly elliptical. This family includes some of the largest living heteropterans, as well as species that are delicate or slender (Schuh \& Slater 1995). The family contains about 267 genera and more than 1884 species worldwide (Henry 2009). The basic suprageneric classification was established by Stål $(1867,1870)$ and Schaefer (1964, 1965), who provided the most comprehensive treatment. Packauskas (1994) gave keys to the subfamilies and tribes of the New World Coreidae and a checklist of published keys to genera and species, and Packauskas (2010) cataloged the New World Coreidae and provided a comprehensive introduction to the literature.

The most comprehensive treatment of the Coreidae for Argentina are by Pennington (1920, 1921) and Kormilev (1954), but only Pennington (1921) dealt with the Acanthocerini.

Many coreids are of great economic importance. As mentioned by Mitchell (2000), grains legumes, rice, cassava, cucurbits, tomatoes, garden vegetables, and various fruit and nut trees are among the crops attacked by coreids worldwide. According to Mitchell (2000), the Acanthocerini includes species of minor economic importance, i.e., Athaumastus haematicus (Stål) that attacks potatoes, cotton, sunflower, oranges and eggplant, Dersagrena flaviventris (Berg) on cotton, and Camptischium clavipes (F.) on castor bean and guaco (Bosq 1937, 1940; Mitchell 2000).

Knowledge of the South American fauna is poor; this is especially true in regard to economically important taxa. Comprehensive keys for identification of the species in the region have not been published.

Most of Argentina lies in the Neotropical faunal region. The country covers an area of 2,791,810 $\mathrm{km}^{2}$ and is bordered by Uruguay, Brazil, Paraguay, Bolivia, and Chile. Approximately $75 \%$ of the country is occupied by arid and semiarid areas, but rainforests are also present in the northeast, i.e., the Yungas and Paranaense regions. Coscarón (submitted) recorded 125 species in 48 genera of Coreidae living from Argentina.

The goal of this paper is to provide an illustrated key to the genera of Acanthocerini (Coreidae) from Argentina (Fig. 38), a diagnosis and redescription of some of its species, geographical distribution, and a list of species for each genus.

\section{Materials and methods}

All specimens from this study are deposited in the collections of the Museo de Ciencias Naturales de La Plata (MLP), La Plata, Buenos Aires, Argentina (http://www.fcnym.unlp. edu.ar/abamuse.html). Photographs were compared with material of the Naturhistoriska Riksmuseet in Stockholm, Sweden (http://www.nrm.se/2.1286b10fdbe80efba80001. html) and the American Museum of Natural History in New York (http://www.amnh. org/). For the geographical distribution we used the program DIVA-GIS 7.1.7 (http:// www.diva-gis.org/) and the distribution of those specimens for which global positioning 
system data were available was used for the construction of maps. Photographs were produced using a Kodak Easy Share (12 megapixels) camera and a magnifying Wild M-Stereomicroscope. The diagnoses of genera are taken from Brailovsky (1987) and O'Shea (1980), except as noted. Coreoidea Species File (Version 1.1/4.1) (http://coreoidea.speciesfile.org/HomePage.aspx) was consulted. All measurement are in millimeters.

\section{Key to the genera of Acanthocerini for Argentina}

1a Pronotum slightly declivent (Fig. 31).................................................. 2

1b Pronotum not slightly declivent.................................... Thlastocoris Mayr

2a Posterior femora tuberculate ventrally and smooth dorsally (Fig. 29) ..........3

2b Posterior femora tuberculate ventrally and dorsally (Fig. 20) ......................5

3a First antennal segment much longer than third segment, humeral angles not sharp (Fig. 30) ....................................................................................... 4

3b First antennal segment much shorter than third segment, humeral angles sharp but hardly produced laterally (Fig. 26) ................................................6

4a Humeral angles of pronotum pointed... Zoreva Amyot \& Serville (Fig. 29)

$4 \mathrm{~b} \quad$ Humeral angles of pronotum rounded.................. Beutelspacoris Brailovsky

5a Dorsal surface of pronotum markedly tuberculate.

Camptischium Amyot and Serville (Fig. 16)

5b Dorsal surface of pronotum punctate but not tuberculate

Crinocerus Burmeister (Fig. 18)

6a Antennifers narrowly but distinctly separated

Dersagrena Kirkaldy (Figs 21, 26)

6b Antennifers meeting mesially Athaumastus Mayr (Figs 1,6)

\section{Taxonomy}

\section{Genus Athaumastus Mayr}

http://species-id.net/wiki/Athaumastus

http://coreoidea.speciesfile.org/Common/basic/Taxa.aspx?TaxonNameID=759

Figs 1, 6

Athaumastus Mayr 1865: 431. Type species: Crinocerus lugens Stål 1855: 184; monotypic.

Diagnosis. (After O`Shea 1980) Body medium-sized, robust, postocular tubercles well developed; antennifers large, placed close together, projecting anteriorly of tylus, with well developed spine on lateral surface; pronotum not very steeply declivent, lateral margins slightly nodulose, humeral angles rounded-angulate, posthumeral, posterior margins relatively smooth; anterior femora with or without distal spines on ventral surface, intermediate and posterior femora armed at least with small apical spines, posterior 


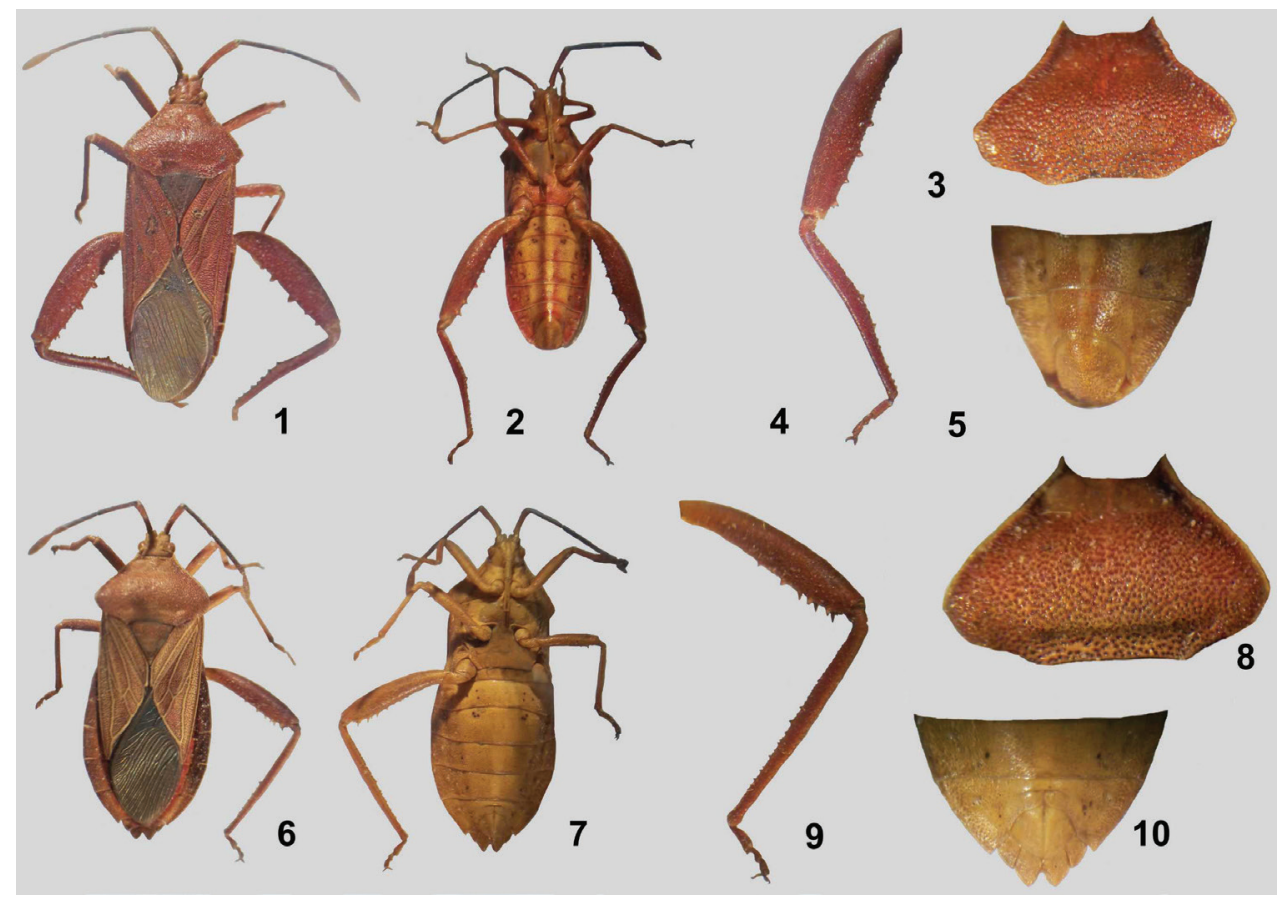

Figures I-10. Athaumastus haematicus (Stål). I-5 $\widehat{\jmath}$, I dorsal view $\mathbf{2}$ ventral view $\mathbf{3}$ pronotum $\mathbf{4}$ hind leg $\mathbf{5}$ male genital capsule, ventral view $\mathbf{6}-\mathbf{1 0}+\mathbf{6}$ dorsal view $\mathbf{7}$ ventral view $\mathbf{8}$ pronotum leg $\mathbf{9}$ hind leg 10 female genital segment, ventral view.

femora markedly curved, incrassate, especially in male; posterior tibiae flattened, armed with teeth along ventral margin, with large tooth halfway along ventral margin in male.

\section{Athaumastus haematicus (Stål)}

http://species-id.net/wiki/Athaumastus_haematicus

http://coreoidea.speciesfile.org/Common/basic/Taxa.aspx?TaxonNameID=779

Figs 1-5, 6-10, 39

Crinocerus haematicus Stål 1859: 455.

Athaumastus haematicus Berg 1878: 85.- Pennington 1920: 13.- Pennington 1921: 36.- Bosq 1937: 113.- Hayward 1942: 72.- Hayward 1960: 30.- Quintanilla et al. 1968: 31.- Viana and Williner 1972: 27.- Quintanilla et al. 1976: 117.- Rizzo 1976: 34.- Viana and Williner 1978: 74.- Quintanilla et al. 1981: 147.

Redescription. Male. $\mathrm{n}=8$. Total body length: 12.3-14.2: head length 1.0-1.2; head width 1.4-0.18; eye width 0.2-0.3; interocular space 0.8-0.9; preocellar distance 0.4; interocellar space 0.2. Rostrum: I 0.7-0.8, II 1.2, III 1.0, IV 0.64-0.7. Antennal seg- 
ments: I 1.8-2.1, II 1.3-2.2, III 2.2-2.7 and IV 1.4-2.0. Pronotum length 2.3-2.9; width 4.6-6.4. Scutellum length 2.3, width 2.2. Length of abdomen with hemelytra: 8.6-9.8; length abdomen with hemelytra: 9.0-10.0; Abdomen width: 3.9-4.6. Dorsal coloration: Head brown tinged with red except antennal segments 2-3, bases of segments with brown tonalities. Pronotum brown, tinged with red. Scutellum dark read. Corium and clavus brown, tinged with red and hemelytral membrane dark brown, veins light brown. Connexival segments dark brown, tinged with red. Ventral coloration: Ground color light brown, tinged with red, mesosternun darker and abdomen not homogeneously dark pigmented. Legs dark brown, tinged with red. Structure: Pronotum rugose. Frontal angles rugose projecting with in as acute projecting spines, humeral angles with two rounded projections. Scutellum granulate. Metafemora with two rows of spines, tibia with small teeth basally. Hemelytra shorter than the abdomen.

Female. $\mathrm{n}=8$. Total body length: 12.0-13.5; head length: 10.0; head width: 1.41.6; eye width: 0.2-0.3; interocular space: 0.8-0.9; preocellar distance: 0.4 ; interocellar space: 0.3. Rostrum: I 0.6-0.8, II 1.2, III 0.9, IV 0.7. Antennal segments: I 1.1-1.7, II 1.5-1.6, III 1.9-20.0 and IV 1.2-1.5. Pronotal length: 2.2-2.7; width: 4.2-5.0. Scutellar length: 1.7; width: 1.5. Length of abdomen with hemelytra: 9.0-11.0; length of abdomen without hemelytra: 9.6-11.3; abdomen width: 4.7-5.4. Dorsally and ventrally light brown. Anterior angles granulate; humeral angles with two rounded projections.

Specimens examined. Argentina: Catamarca: $10^{\lambda}$ Andalgalá $\left(27^{\circ} 36^{\prime} 02^{\prime \prime S}\right.$,

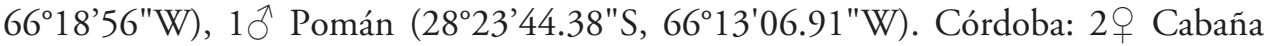

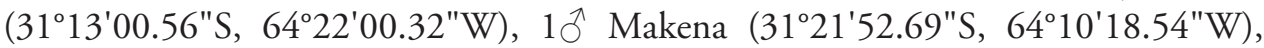

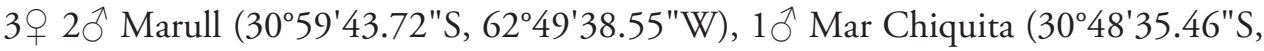
$\left.62^{\circ} 52^{\prime} 31.68^{\prime \prime W}\right), 10^{\Uparrow}$ Rayo cortado $\left(30^{\circ} 04^{\prime} 26.09^{\prime \prime S}, 63^{\circ} 49^{\prime} 25.99^{\prime \prime W}\right) .2930^{\Uparrow}$ Corrientes. Chaco: 1 \% Charata $\left(27^{\circ} 13^{\prime} 06.31^{\prime \prime S}, 61^{\circ} 11^{\prime} 15.89^{\prime \prime W}\right)$, 1 ㅇ El zapallar

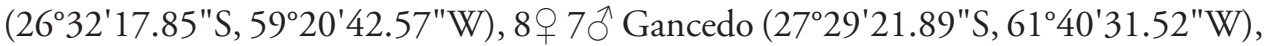
1 우 Resistencia $\left(27^{\circ} 27^{\prime} 05^{\prime \prime} \mathrm{S}, 58^{\circ} 59^{\prime} 10^{\prime \prime W}\right)$. Formosa: $20^{\Uparrow}$ Ingeniero Juárez $\left(23^{\circ} 53^{\prime} 46.85^{\prime \prime S}, 61^{\circ} 51^{\prime} 37.88^{\prime \prime W}\right) .1{ }^{\Uparrow}$ La Rioja. 1 ㅇ San Luis. Santiago del Estero: $1{ }^{\lambda}$ Río Salado (27\%44'32.51"S, 64²1'16.97"W).

Distribution. Argentina: Buenos Aires; Catamarca; Córdoba: Carlos Paz, El Sauce, San Javier; Corrientes: Alvear Department, Bella Vista Department, Berón de Astrada Department, Capital Department, Colón Department, Concepción Department, Concordia Department, Diamante Department, Empedrado Department, Esquina Department, General Paz Department, La Paz Department, Mburucuyá Department, Paraná Department, Paso de los Libres Department, Saladas Department, San Martín Department, San Miguel Department, San Roque Department, Santo Tomé Department, Victoria Department; Chaco; Entre Ríos: Concordia; Formosa; Jujuy; La Pampa; La Rioja; Mendoza; Misiones: Alem Department, Apóstoles Department, Cainguás Department, Concepción de la Sierra Department, Guaraní Department, Montecarlo Department, Oberá Department, San Javier Department, San Martín Department, 25 de Mayo Department; Neuquén; Salta; San Juan; San Luis: San Gerónimo, Suyuque; Santa Fé; Santiago del Estero; Tucumán. 


\section{Athaumastus macer Brailovsky}

http://coreoidea.speciesfile.org/Common/basic/Taxa.aspx?TaxonNameID=781

Athaumastus macer Brailovsky 1993:115.

Distribution. Argentina: Tucumán

\section{Athaumastus subcarinatus (Stål)}

http://species-id.net/wiki/Athaumastus_subcarinatus

http://coreoidea.speciesfile.org/Common/basic/Taxa.aspx?TaxonNameID=783

Figs 11-14, 40

Crinocerus subcarinatus Stål 1859: 455.

Athaumastus subfoveolatus Berg 1892: 66.-Coscarón 1998: 4.

Athaumastus subcarinatus Pennington 1920: 13.- Pennington 1921: 38.- Quintanilla et al. 1968: 31.- Quintanilla et al. 1976: 118.Viana and Williner 1978: 74.

Redescription. Male: $\mathrm{n}=8$. Total body length: 12.4-13.0; head length: 0.8-1.1; head width: 1.4-1.5; eye width: $0.2-0.3$; interocular space: 0.8 ; preocellar distance: 0.3 ; interocellar space: 0.2 . Rostrum: I 0.4-0.7, II 1.1, III 0.7, IV 0.4-0.6. Antennal segments: I 1.3-1.7, II 1.5-1.9, III 1.4-2.1 and IV 1.3. Pronotum length: 2.2-2.7; width: 3.4-3.9. Scutellum length: 1.4, wide 1.6. Length of abdomen with hemelytra: 8.0; length of abdomen with hemelytra: 9.0; abdomen width: 4.0-4.1. Dorsal coloration: Head including antennal segments 1-3 brown, 4 dark brown. Pronotum brown, margins light brown. Scutellum and corium brown tinged with dark red, hemelytral membrane dark brown. Connexival segments light brown. Ventral coloration: Ground color brown with following areas dark brown: mesosternun, metapleura, and abdomen not homogeneously dark brown. Fore- and middle legs light brown, hind leg dark brown. Structure: head granulate brown; antennal segment IV pilose; pronotum granulate. Frontal angles rugose, with a projecting as acute spines; humeral angles with two rounded projections. Scutellum granulate, dark brown. Metafemora with two rows of 6 spines; tibia with small teeth basally. Hemelytra granulated brown tinged with dark red, shorter than abdomen.

Specimens examined. Argentina: Buenos Aires: 1 + Junín $\left(34^{\circ} 35^{\prime} 16.37^{\prime \prime S}\right.$, $\left.60^{\circ} 58^{\prime} 45.71 " \mathrm{~W}\right)$. Córdoba: 1 ㅇ Villa Nueva $\left(32^{\circ} 26^{\prime} 08.91 " \mathrm{~S}, 63^{\circ} 14^{\prime} 59.39^{\prime \prime W}\right)$. For-

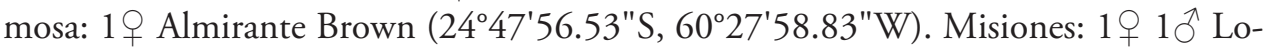
reto $\left(27^{\circ} 18^{\prime} 59.85^{\prime \prime} \mathrm{S}, 55^{\circ} 32^{\prime} 00.04^{\prime \prime} \mathrm{W}\right)$.

Distribution. Argentina: Córdoba: Carlos Paz, San Javier; Corrientes: Capital Department, Lavalle Department; Entre Ríos: Concordia Department, Federación Department, Gualeguachú Department, Paraná Department, Villaguay Department; Salta.

Remarks. These are the first records of this species from Buenos Aires, Formosa, and Misiones. 


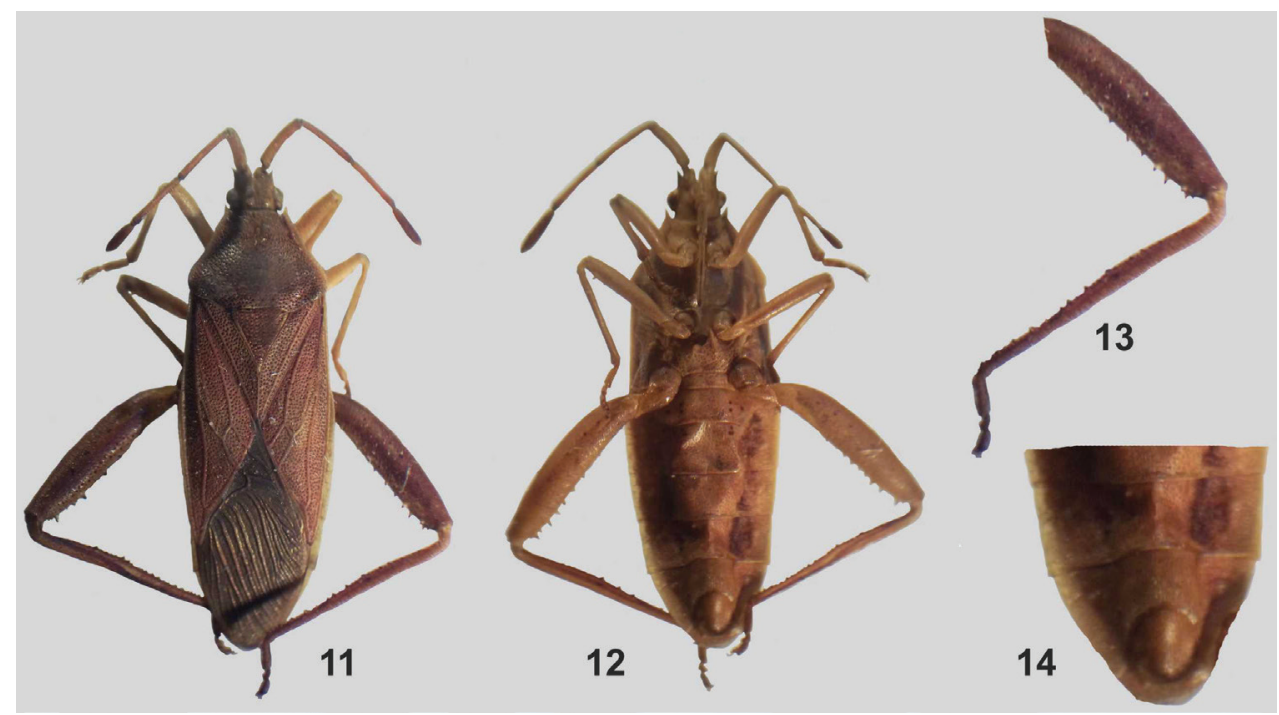

Figures I I-| 4. Athaumastus subcarinatus (Stål) ô. $\mathbf{1} \mathbf{2}$ dorsal view $\mathbf{1} \mathbf{2}$ ventral view $\mathbf{3}$ hind leg $\mathbf{1} \mathbf{4}$ male genital capsule, ventral view.

\section{Athaumastus subterlineatus Bergroth}

http://coreoidea.speciesfile.org/Common/basic/Taxa.aspx?TaxonNameID=785

Athaumastus subterlineatus Bergroth 1912: 85

Distribution. Argentina: Santiago del Estero, Río Salado.

\section{Genus Beutelspacoris Brailovsky}

http://species-id.net/wiki/Beutelspacoris

http://coreoidea.speciesfile.org/Common/basic/Taxa.aspx?TaxonNameID=760

Beutelspacoris Brailovsky 1987: 523. Type species: Beutelspacoris sanchezi Brailovsky 1987: 524 .

Moreyacoris Casini 1989: 25. Type species: Moreyacoris dilatata Casini 1989 : 26.

Diagnosis. (After Brailovsky 1987) Body relatively small, robust, postocular tubercles prominent; antenniferous tubercles large. Pronotum slightly declivent, wider than long; callar region distinct; collar narrow; humeral angles rounded. All femora at least slightly incrassate; posterior femora more incrassate; all femora with subdistal spines on ventral surface and dorsally smooth; anterior and intermediate tibiae terete, sulcate, and unarmed; posterior tibiae slightly flattened, armed with small teeth along internal margin. 


\section{Beutelspacoris sanchezi Brailovsky}

http://coreoidea.speciesfile.org/Common/basic/Taxa.aspx?TaxonNameID=788

Beutelpacioris sanchezi Brailovsky 1987: 524.

Distribution. Argentina: Santiago del Estero: Lago Muyo.

\section{Beutelspacoris dilatata Casini}

http://coreoidea.speciesfile.org/Common/basic/Taxa.aspx?TaxonNameID=787

Moreyacoris dilatata Casini 1989: 26.- Bachmann 1999: 221.

Beutelspacoris dilatata Brailovsky and Barrera 2003: 888.

Distribution. Argentina: Jujuy; La Rioja: Nanogasta.

\section{Genus Camptischium Amyot \& Serville}

http://species-id.net/wiki/Camptischium

http://coreoidea.speciesfile.org/Common/basic/Taxa.aspx?TaxonNameID=761

Fig. 15

Camptischium Amyot and Serville 1843: 213. Type species: Camptischium spinosum Amyot and Serville 1843: 213; monotypic.

Diagnosis. (After O'Shea 1980) Body-medium sized, robust, stout, elliptical or obovate; postocular tubercles relatively small, antennifers large with marked external spine; pronotum steeply declivent, humeral angles produced laterally into a sharp spine, posthumeral margins nodulose; all femora somewhat incrassate, armed with spines at least distally on ventral surface, posterior femora markedly curved, incrassate, especially in male, with tubercles ventrally and dorsally; posterior tibiae flattened, with spines situated about equidistant from anterior and lateral margins.

\section{Camptischium clavipes (Fabricius)}

http://species-id.net/wiki/Camptischium_clavipes

http://coreoidea.speciesfile.org/Common/basic/Taxa.aspx?TaxonNameID=1490

Figs 15-17, 41

Coreus clavipes Fabricius 1803:196.

Acanthocerus (Camptischium) clavipes Berg 1878: 83.- Pennington 1921: 35.

Camptischium clavipes Pennington 1920: 13.- Viana and Williner 1978: 75. 


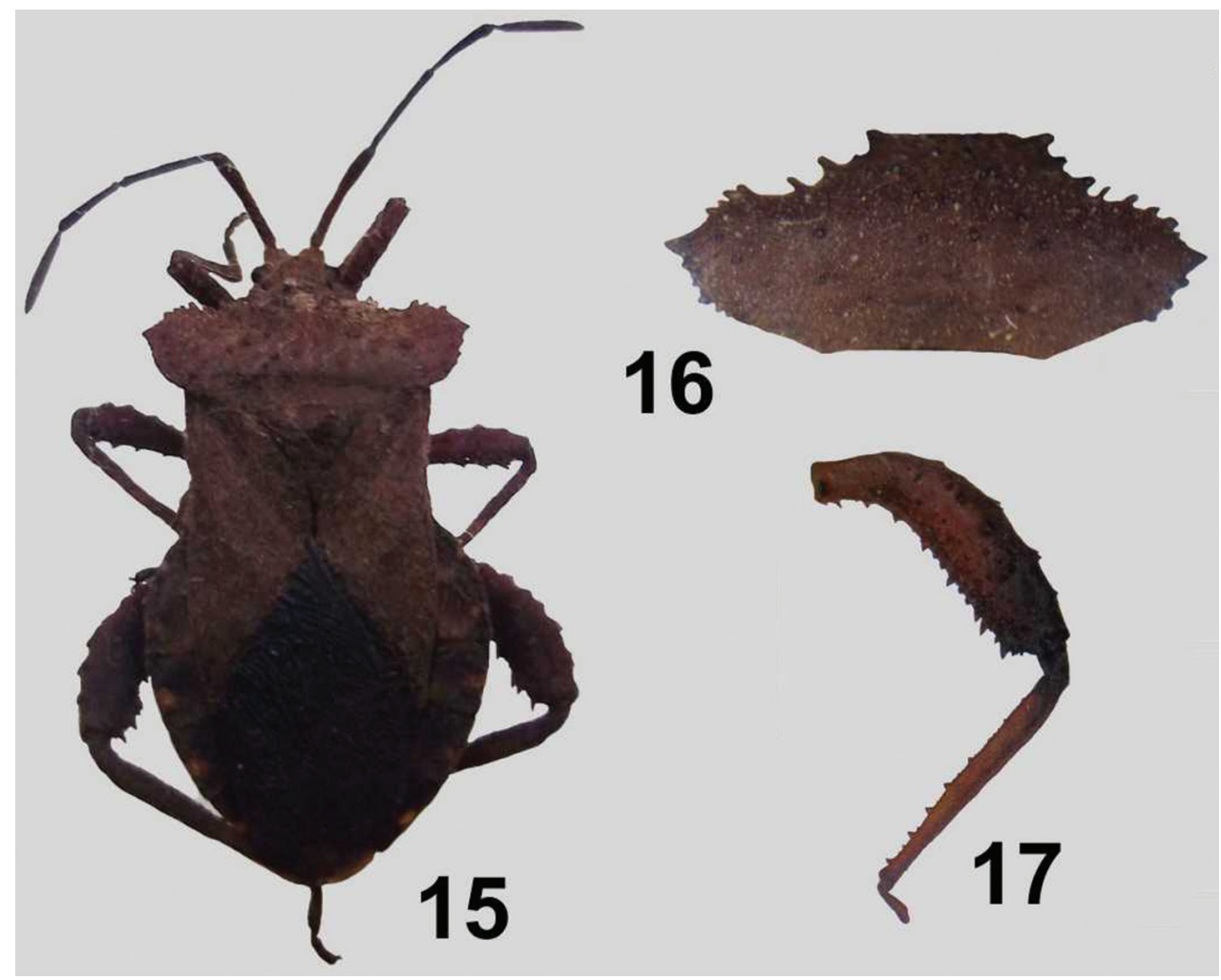

Figures 15-17. Camptischium clavipes (Fabricius) $\widehat{~}$. $\mathbf{1 5}$ dorsal view $\mathbf{1 6}$ pronotum $\mathbf{1 7}$ hind leg.

Acanthocoris clavipes Blöte 1935: 220.

Acanthocerus clavipes Bosq 1940: 401.- Hayward 1960: 30.- Quintanilla et al. 1981: 147. Captischium clavipes Viana and Williner 1972: 27.

Specimens examined. Argentina: Buenos Aires: 2 q La Plata (34 ${ }^{\circ} 55^{\prime} 16^{\prime \prime S}$, $\left.57^{\circ} 57^{\prime} 17^{\prime \prime W}\right), 1 q$ Pereyra Iraola $\left(34^{\circ} 50^{\prime} 44.08^{\prime \prime S}, 58^{\circ} 10^{\prime} 42.94^{\prime \prime W}\right) .192 \delta^{\lambda}$ Catamarca. Córdoba: 19 Bella vista $\left(29^{\circ} 32^{\prime} 07.71^{\prime \prime S}, 64^{\circ} 10^{\prime} 02.87^{\prime \prime W}\right), 1 \sigma^{\lambda}$ Villa

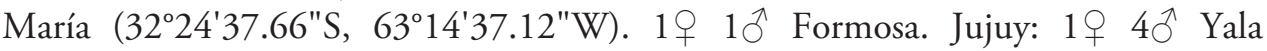
$\left(24^{\circ} 07^{\prime} 10.78^{\prime \prime S}, 65^{\circ} 24^{\prime} 06.78^{\prime \prime W}\right), 1$ ㅇ Ledesma (23⒋ $\left.48.79^{\prime \prime S}, 64^{\circ} 47^{\prime} 41.47^{\prime \prime W}\right)$, $1 \delta^{\Uparrow}$ Reyes $\left(24^{\circ} 09^{\prime} 49.08^{\prime \prime S}, 6^{\circ} 22^{\prime} 42.99^{\prime \prime W}\right)$. Salta: 2 ㅇ $1 \delta^{\Uparrow}$ San Lorenzo $\left(26^{\circ} 06^{\prime} 34^{\prime \prime S}\right.$, 64³8'34"W). 3ㅇ 40̊ Tucumán.

Distribution. Argentina: Catamarca; Córdoba: Carlos Paz, Río San José; Chaco; Formosa; Jujuy; La Rioja; Mendoza; Misiones: Corpus, Department Cainguás, Department Montecarlo; Neuquén; Salta; San Juan; Tucumán.

Remarks. This is the first record of this species from Buenos Aires. 


\section{Genus Crinocerus Burmeister}

http://species-id.net/wiki/Crinocerus

http://coreoidea.speciesfile.org/Common/basic/Taxa.aspx?TaxonNameID=763

Fig. 18

Crinocerus Burmeister 1835: 318. Type species: Cimex sanctus Fabricius 1775: 709, by designation (O'Shea 1973).

Diagnosis. (After O'Shea, 1980) Body medium-sized, robust, oblong; postocular tubercles well developed; antennifers large, pronounced, situated close together with distinct external spine; pronotum steeply declivent, posterior angles rounded; all femora armed at least with subdistal spines on ventral surface, posterior femora curved, incrassate especially in male, armed with tubercles on all surfaces, becoming spines on ventral surface; posterior tibiae flattened, straight in female, curved in male.

\section{Crinocerus sanctus (Fabricius)}

http://species-id.net/wiki/Crinocerus_sanctus http://coreoidea.speciesfile.org/Common/basic/Taxa.aspx?TaxonNameID=792

Figs 18-20, 42

Cimex sanctus Fabricius 1775: 709.

Crinocerus sanctus Berg 1878: 82.- Pennington 1920: 13.- Pennington 1921: 35.Bosq 1937: 115.- Quintanilla et al. 1981: 148.

Specimens examined. Argentina: Misiones: 2 ㅇ $30^{\wedge}$ Loreto $\left(27^{\circ} 18^{\prime} 59.85^{\prime \prime} \mathrm{S}\right.$, $\left.55^{\circ} 32^{\prime} 00.04 " \mathrm{~W}\right), 3$ + $10^{\lambda}$ San Ignacio $\left(27^{\circ} 15^{\prime} 34.49^{\prime \prime} \mathrm{S}, 55^{\circ} 32^{\prime} 19.23^{\prime \prime W}\right)$. Tucumán: 1 q.

Distribution. Argentina: Buenos Aires; Misiones: Corpus, DepartmentAlem, Department Cainguás, Department El dorado, Department Guaraní, Department Iguazú, Department San Ignacio, Department San Javier, Santa Ana.

Remarks. This is the first record of this species from Tucumán.

\section{Genus Dersagrena Kirkaldy}

http://species-id.net/wiki/Dersagrena

http://coreoidea.speciesfile.org/Common/basic/Taxa.aspx?TaxonNameID=764

Figs $21-26$

Dalcera Signoret 1863: 556. Type species: Dalcera lacerdae Signoret 1863, monotypic. Dersagrena Kirkaldy 1904: 280. New name for Dalcera Signoret, takes the type for that genus.

Diagnosis. (After O'Shea 1980) Body relatively small, narrow; postocular tubercles prominent, antennifers large, situated close together, pronotum relatively shallowly 


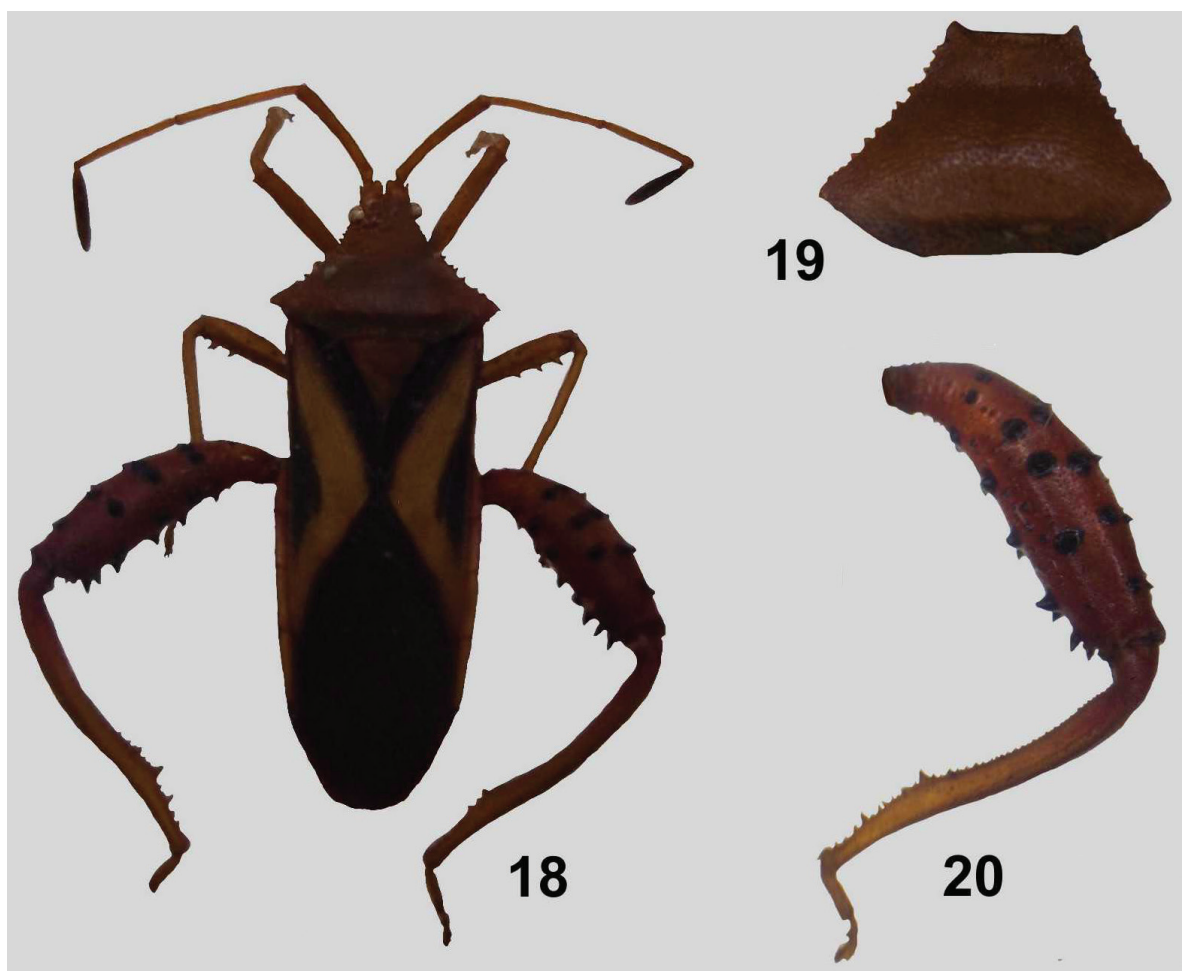

Figures 18-20. Crinocerus sanctus (Fabricius) ô. 18 dorsal view 19 pronotum, 20 hind leg.

declivent, lateral margins slightly nodulose, humeral angles sharp, posthumeral, posterior margins smooth; anterior femora unarmed, intermediate femora armed distally on ventral surface with small spines, at least in male, posterior femora incrassate, armed with spines on ventral surface, especially in male, posterior tibiae flattened with small teeth on ventral margin, more in the male.

\section{Dersagrena flaviventris (Berg)}

http://species-id.net/wiki/Dersagrena_flaviventris

http://coreoidea.speciesfile.org/Common/basic/Taxa.aspx?TaxonNameID=793

Figs 21-25, 43

Dalcera flaviventris Berg 1879: 282.- Lethierry and Severin 1894: 22.

Athaumastus flaviventris Pennington 1920: 13.- Pennington 1921: 38.- Bosq 1937: 113. - Coscarón 1998: 2.

Dersagrena flaviventris Mitchell 2000: 384.

Redescription. Holotype. Female. $\mathrm{n}=1$. Total body length: 10.0; head length: 1.2; head width: 1.5 ; eye width: 0.3 ; interocular space: 0.7 ; preocellar distance: 0.2 ; interocellar space: 0.3 . Rostrum: I 1.0, II 1.2, III 1.0, IV 0.8. Antennal segments length: I 


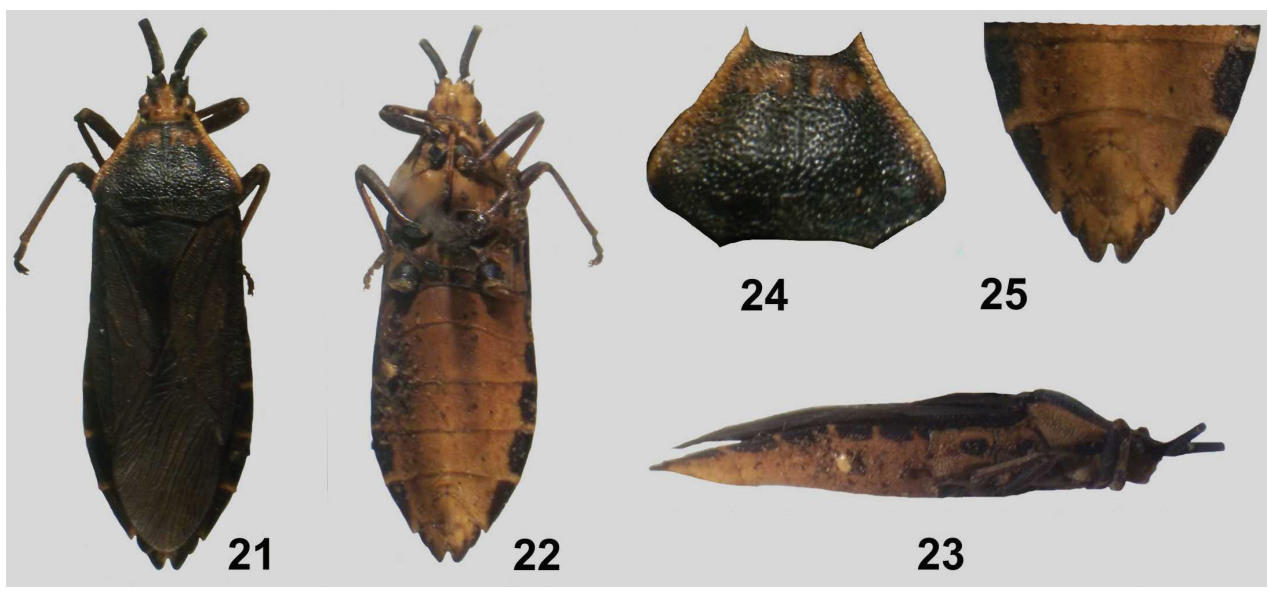

Figures 2I-25. Dersagrena flaviventris (Berg) ${ }^{+} .2 \mathbf{~ I ~ d o r s a l ~ v i e w ~} \mathbf{2 2}$ ventral view $\mathbf{2 3}$ lateral view $\mathbf{2 4}$ pronotum 25 genital segments, ventral view.

1.1, II 1.6, 1.7 III and IV (segment missing). Pronotal length: 2.8; width: 4.1. Scutellar length: 1.4; width 1.3. Length of abdomen with male hemelytra: 9.6; length of abdomen with hemelytra: 10.0; abdomen width: 4.7. Dorsal coloration: Head dark brown, light brown interocular space. Pronotum dark brown except two stains on anterior region and lateral margins light brown. Scutellum, coria, and hemelytral membrane dark brown. Connexival segments dark brown with intersegmental line light brown. Ventral coloration: Ground color light brown, connexival segments dark brown. Legs dark brown. Structure: pronotum granulate; frontal angles granulate and projecting as acute spines reaching ocular tubercles; humeral angles with two rounded projections; scutellum granulate. Hemelytra shorter than the abdomen.

Specimen examined. Argentina: Córdoba: 19 Alta Gracia $\left(31^{\circ} 24^{\prime} 53.38^{\prime \prime S}\right.$, $64^{\circ} 10^{\prime} 36.61^{\prime \prime W)}$.

Distribution. Argentina: Buenos Aires, Córdoba: Río Cuarto; Chaco; San Luis: Villa Mercedes; Santiago del Estero; Tucumán.

\section{Dersagrena lacerdae (Signoret)}

http://coreoidea.speciesfile.org/Common/basic/Taxa.aspx?TaxonNameID=794

Dalcera lacerdae Signoret 1863: 556.

Dersagrena lacerdae Pennington 1920: 14.- Pennington 1921: 39.

Distribution. Argentina: Catamarca; Chaco; Formosa; Jujuy; La Rioja; Mendoza; Misiones; Neuquén; San Juan, Tucumán. 


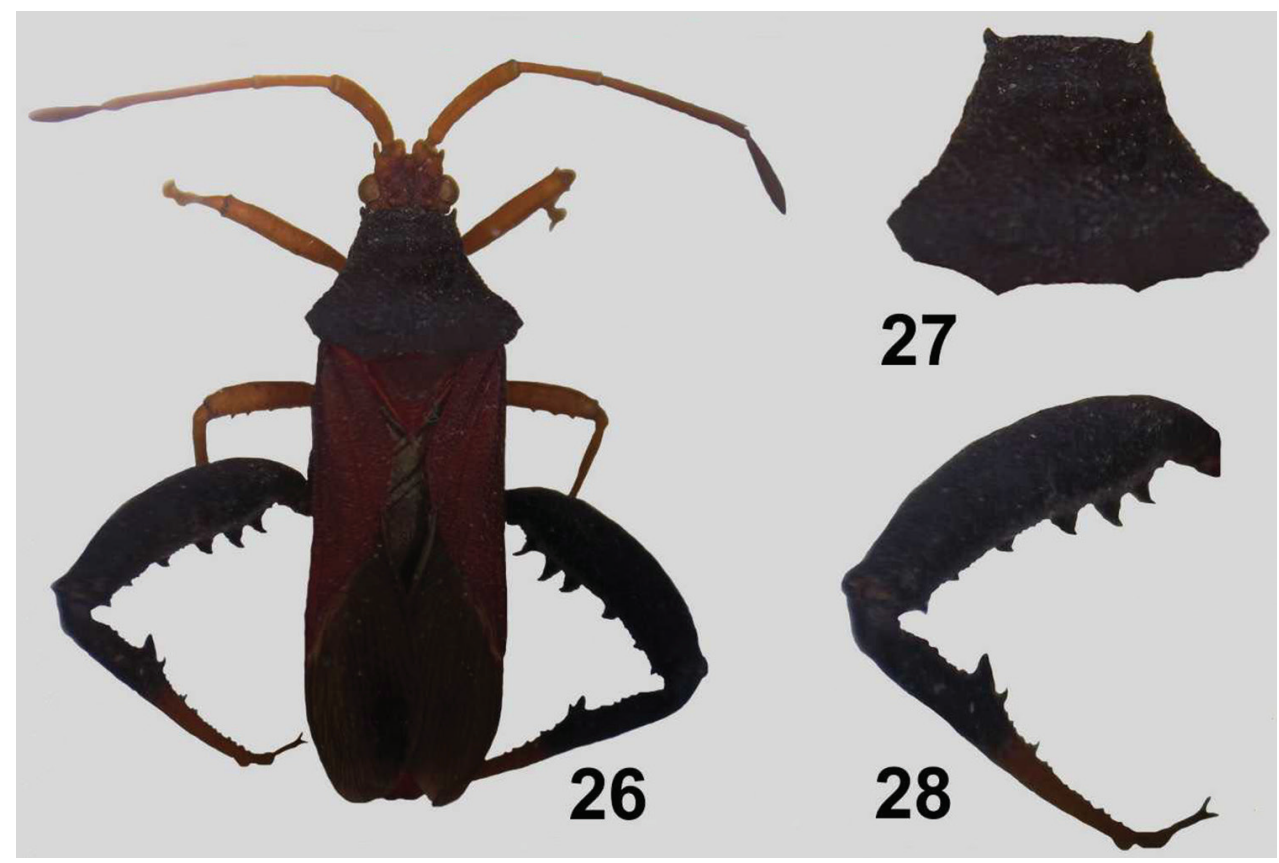

Figures 26-28. Dersagrena subfoveolata (Berg) $\curvearrowright .26$ dorsal view $\mathbf{2 7}$ pronotum $\mathbf{2 8}$ hind leg.

\section{Dersagrena subfoveolata (Berg)}

http://species-id.net/wiki/Dersagrena_subfoveolata http://coreoidea.speciesfile.org/Common/basic/Taxa.aspx?TaxonNameID=795

Figs 26-28, 34-37, 44

Athaumastus subfoveolatus Berg 1892: 66.- Bachmann 1999: 197.

Dersagrena subfoveolatus Pennington 1920: 14.- Pennington 1921: 39.

Desagrena subflaveolatus Viana and Williner 1978: 75.

Specimens examined. Argentina: Chaco: 19 Corzuela $\left(26^{\circ} 57^{\prime} 21.84 " \mathrm{~S}\right.$, $\left.60^{\circ} 58^{\prime} 14.21^{\prime \prime W}\right), 10^{\top}$ Gancedo $\left(27^{\circ} 29^{\prime} 21.89 " \mathrm{~S}, \quad 61^{\circ} 40^{\prime} 31.52^{\prime \prime W}\right)$. Formosa: $1 \delta^{\lambda}$ Ibarreta $\left(25^{\circ} 12^{\prime} 55.21 " \mathrm{~S}, 59^{\circ} 51^{\prime} 27.69^{\prime \prime} \mathrm{W}\right)$. La Rioja: $10^{\lambda}$ Nonogasta $\left(29^{\circ} 18^{\prime} 11.07^{\prime \prime S}, 67^{\circ} 30^{\prime} 05.67^{\prime \prime W}\right)$. Salta: 1 ㅇ $40^{\lambda}$ Talapampa $\left(25^{\circ} 32^{\prime} 00.01^{\prime \prime S}\right.$,

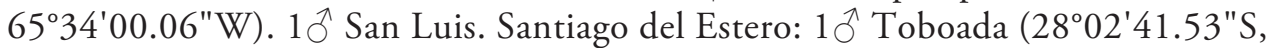
$63^{\circ} 47^{\prime} 16.89^{\prime \prime W)}$.

Distribution. Argentina: Catamarca; Córdoba: Capilla del Monte; Jujuy; La Pampa; La Rioja; Mendoza; Neuquén; Salta; San Juan; San Luis; Santa Fé; Santiago del Estero; Tucumán.

Remarks. These are the first records of this species from Chaco and Formosa. 


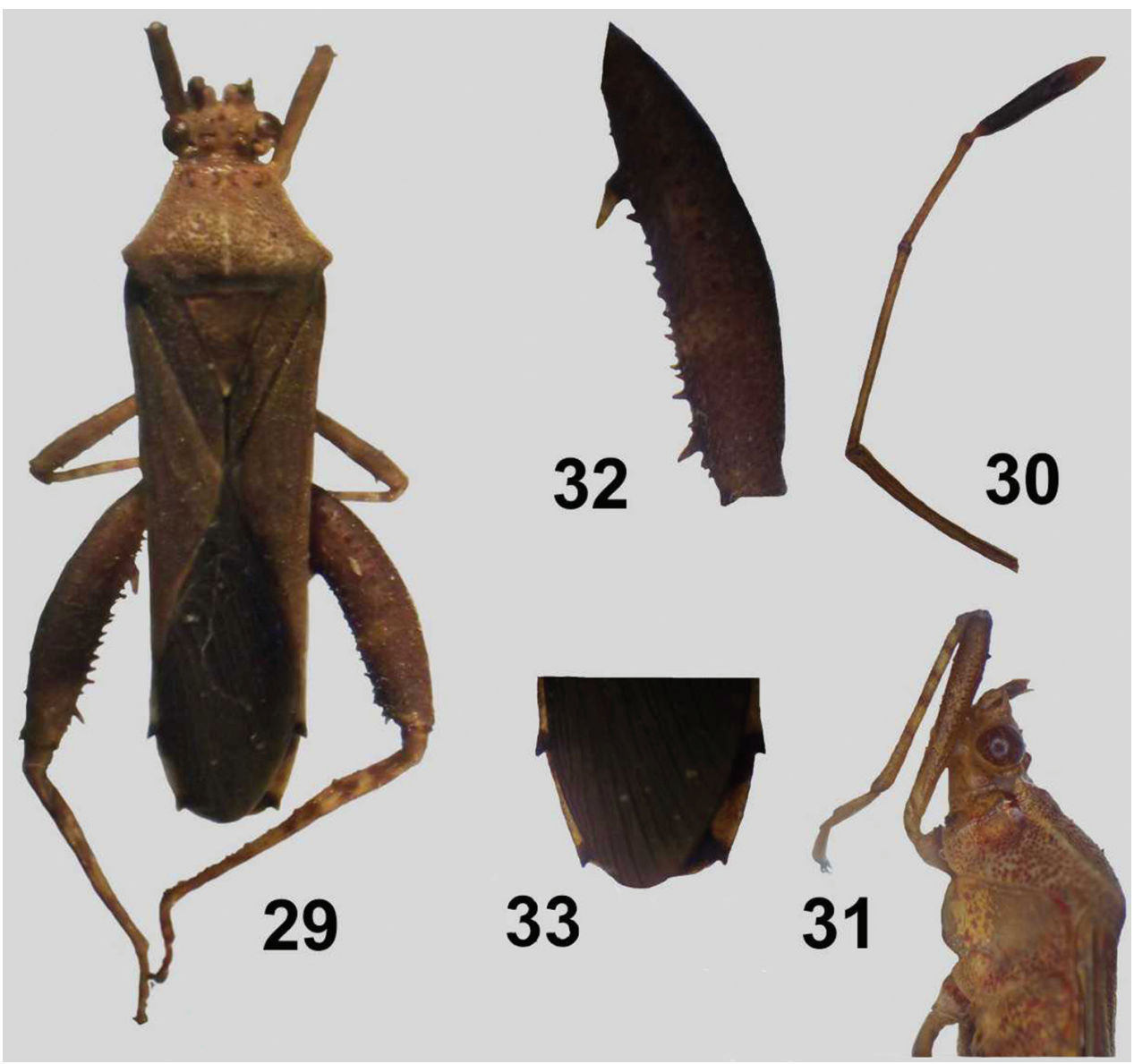

Figures 29-33. Zoreva dentipes Fabricius $\partial$ $\widehat{2} \mathbf{2 9}$ dorsal view $\mathbf{3 0}$ antenna $\mathbf{3} \mathbf{I}$ head and pronotum $\mathbf{3 2}$ hind femur 33 abdomen dorsal view.

\section{Genus Thlastocoris Mayr}

http://species-id.net/wiki/Thlastocoris

http://coreoidea.speciesfile.org/Common/basic/Taxa.aspx?TaxonNameID=774

Thlastocoris Mayr 1866: 88. Type species: Thlastocoris laetus Mayr, monotypic.

Diagnosis. (After O`Shea 1980) Body relatively small, postocular tubercles well developed, antennifers large, fairly widely separated, external spine present on antennifers; pronotum rather shallowly declivent; all femora at least slightly incrassate, posterior femora more incrassate, a little more in male than females, anterior, intermediate femora smooth, or with obsolete spines distally on ventral surface, posterior femora with spines on ventral surface; posterior tibiae of female straight, slightly flattened, of male more flattened with widest part at midpoint, armed with teeth along ventral margin. 


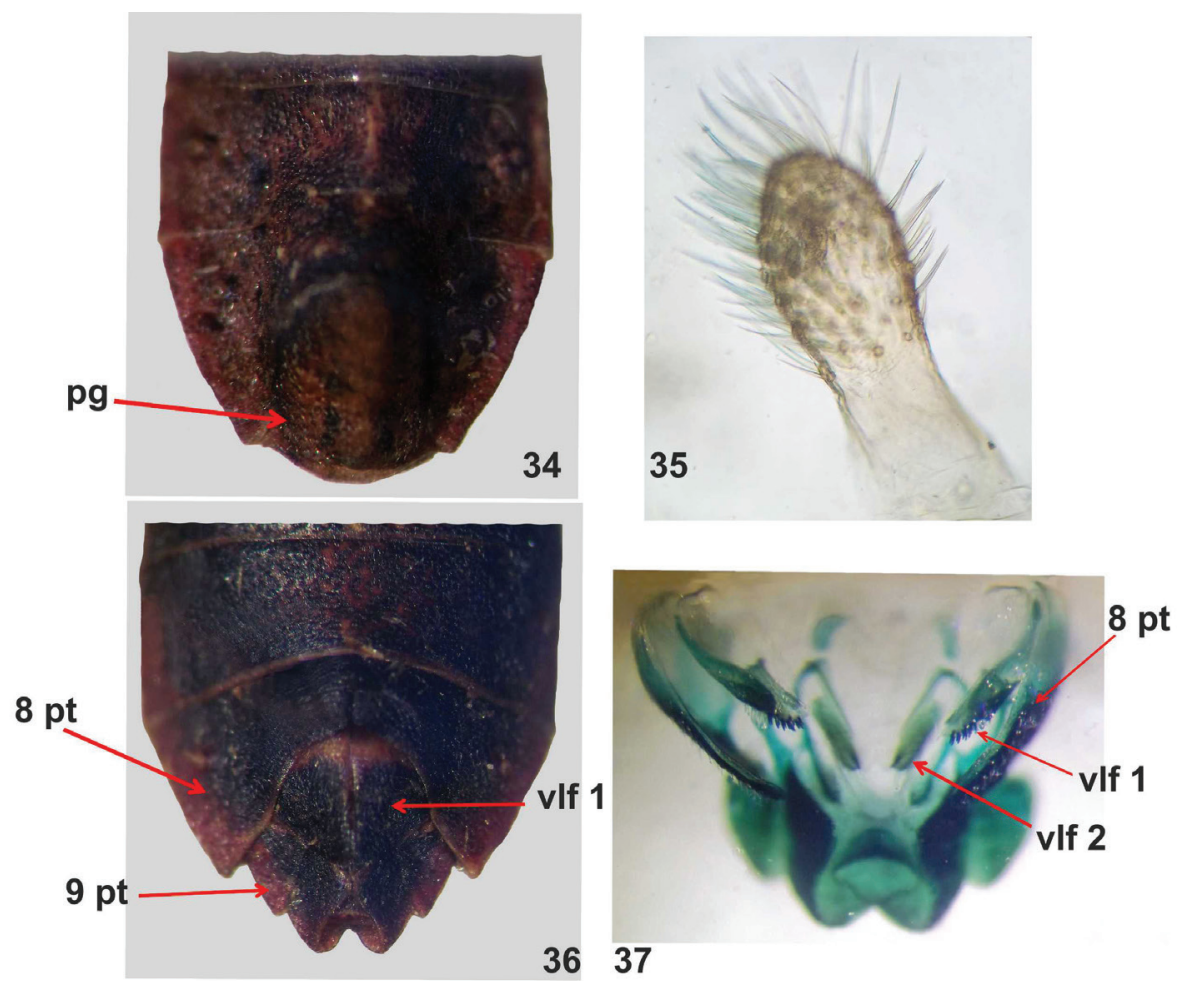

Figures 34-37. Dersagrena subfoveolata (Berg) $\widehat{~}$, $q$. 34-35 male genital capsule, ventral view 34 pg: pygophore 35 paramere 36-37 female genital segments, ventral view 368 pt: paratergite eight, 9 pt: paratergite nine, vlf. 1: first valvifer 378 pt: paratergite eight, vlf. 1: first valvifer, vlf. 2: second valvifer.

\section{Thlastocoris hernandezi Brailovsky}

http://coreoidea.speciesfile.org/Common/basic/Taxa.aspx?TaxonNameID=2210

Thlastocoris hernandezi Brailovsky 1990: 108.

Distribution. Argentina: Formosa: Gran Guardia.

\section{Genus Zoreva Amyot \& Serville}

http://species-id.net/wiki/Zoreva

http://coreoidea.speciesfile.org/Common/basic/Taxa.aspx?TaxonNameID=775

Fig. 29

Zoreva Amyot and Serville 1843: 216. Type species: Coreus dentipes Fabricius 1803: 196.

Diagnosis. (After O`Shea, 1980) Body narrow and elongated. Pronotum almost hexagonal, humeral angle developed into a long sharp spine of variable length and di- 

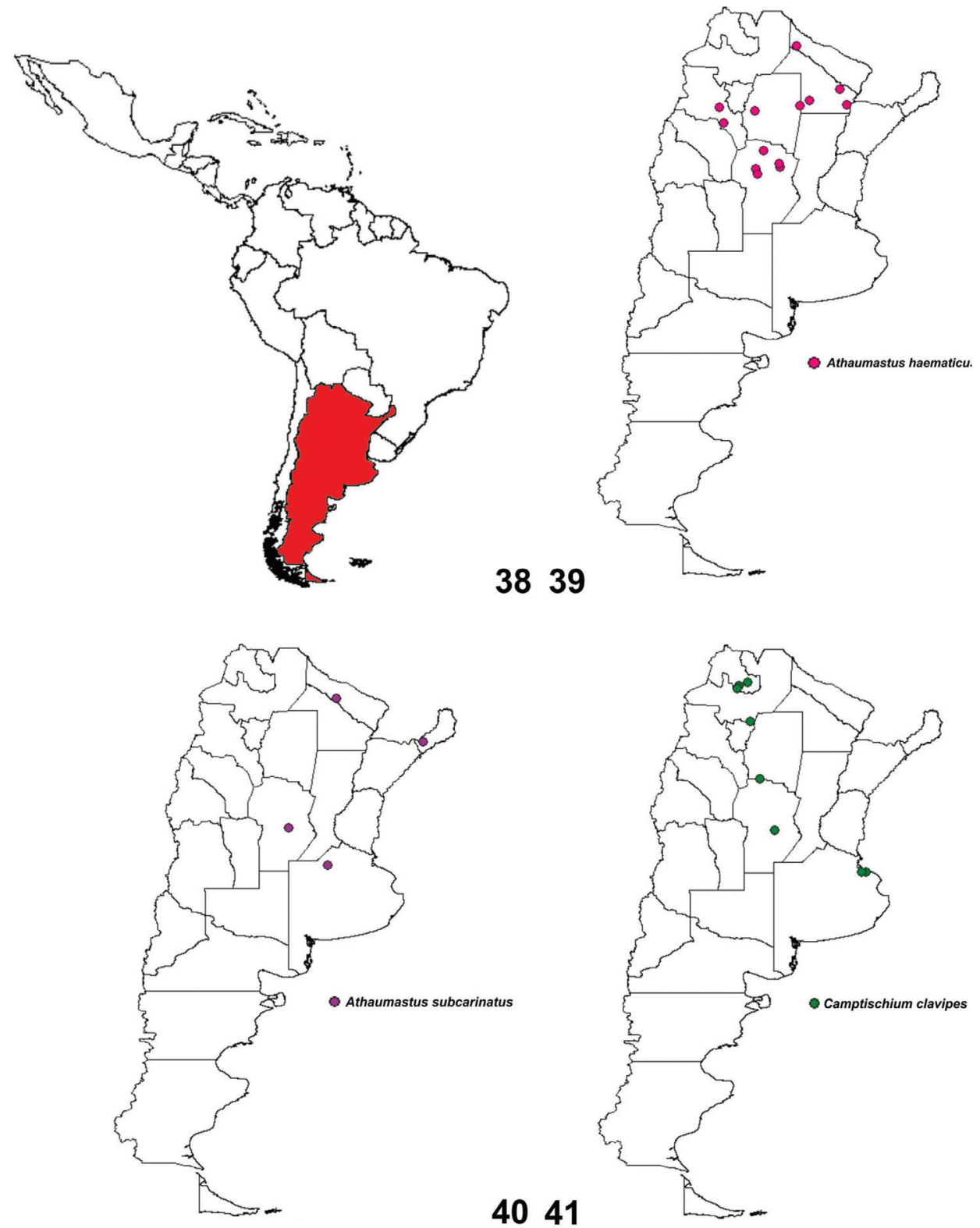

3839

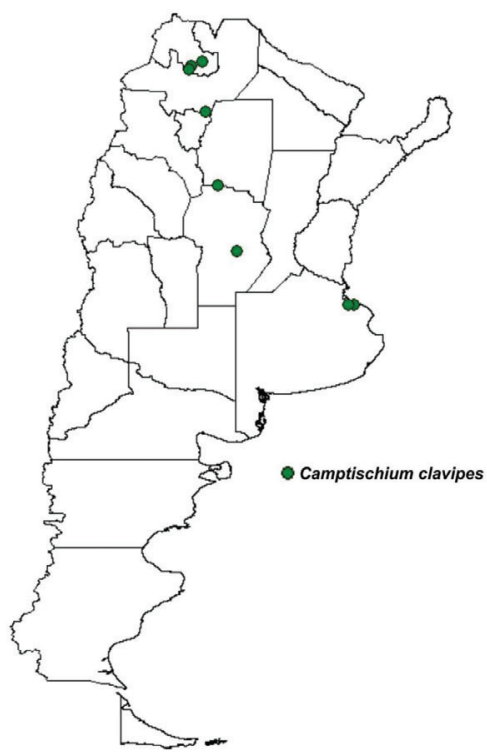

Figure 38-4I. Geographical distribution: 38 Argentina geographical location 39 Athaumastus haematicus (Stål) 40 Athaumastus subcarinatus (Stål) 4 I Camptischium clavipes (Fabricius).

rection, posthumeral edge with small conspicuous teeth, posterior border straight or slightly concave. Leg dimorphic. Males: Femora ventrally armed with spines of variable length, femur anterior generally smooth texture; hind femur swollen and curved. Females: Femora armed with spines ventrally, length variable anterior and middle femora thinner and a little more drawn back, but never curved. 

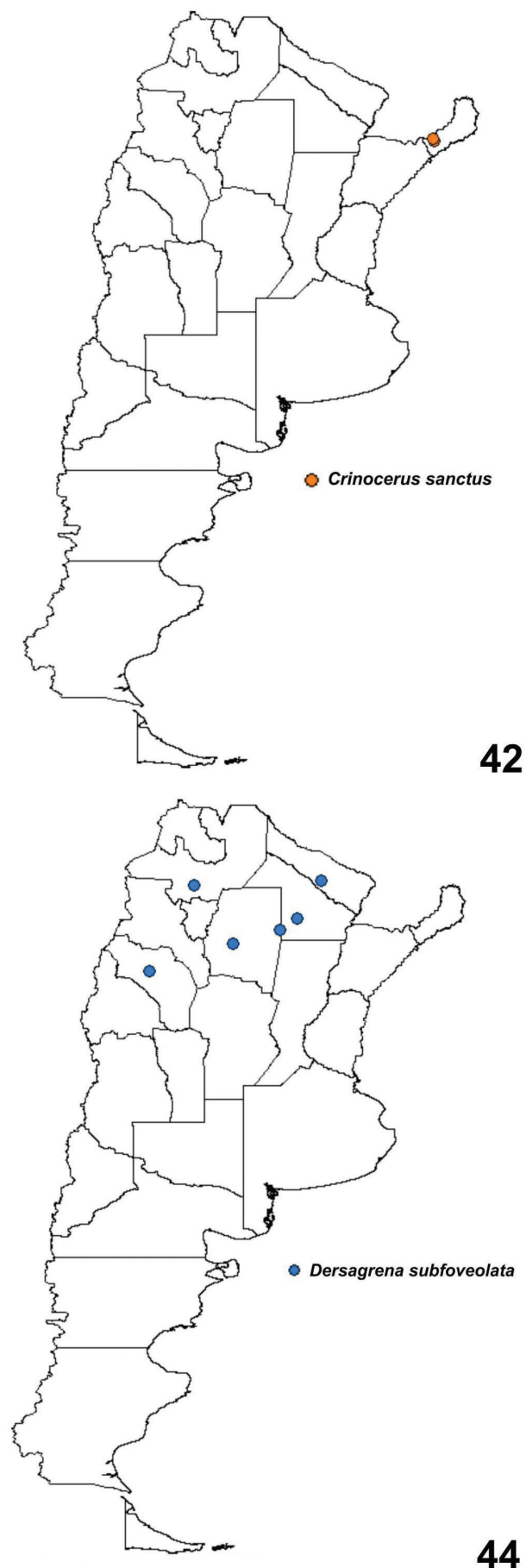

4243
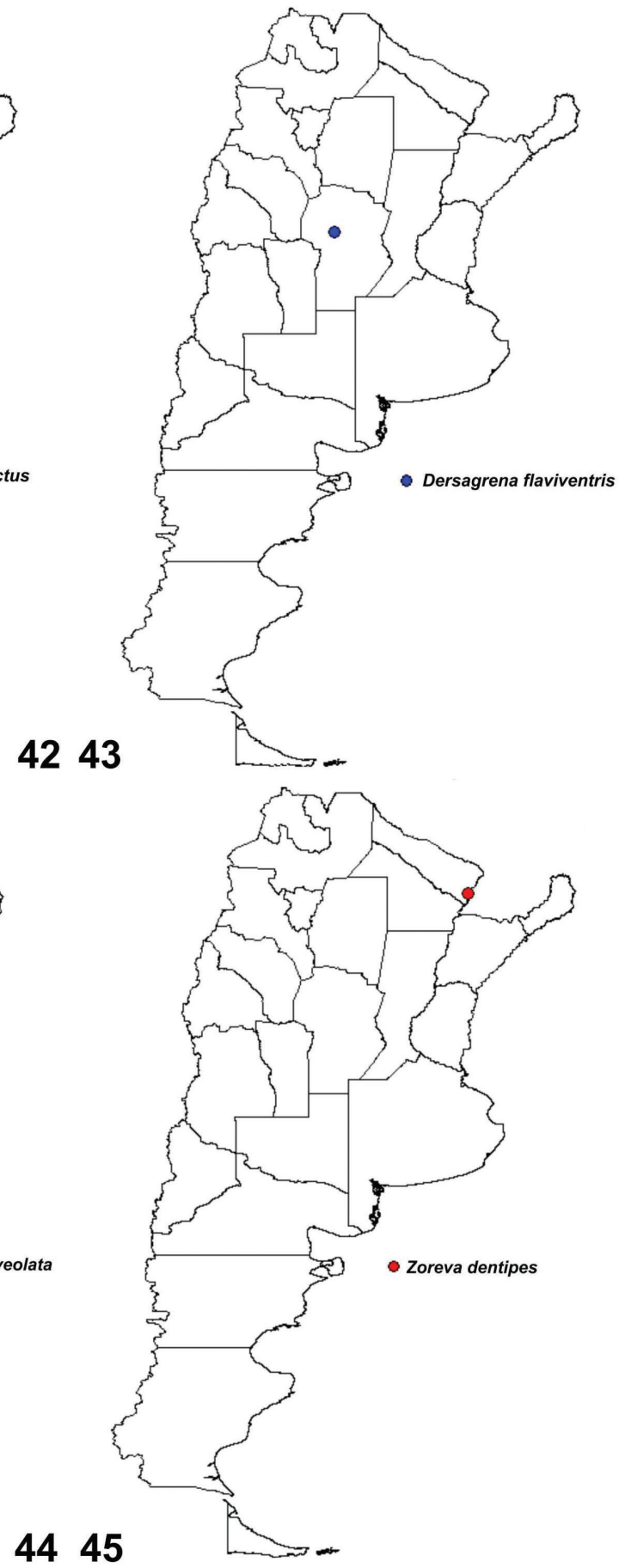

Figure 42-45. Geographical distribution: 42 Crinocerus sanctus (Fabricius), 43 Dersagrena flaviventris (Berg) 44 Dersagrena subfoveolata (Berg) 45 Zoreva dentipes Fabricius. 


\section{Zoreva dentipes Fabricius}

http://species-id.net/wiki/Zoreva_dentipes

http://coreoidea.speciesfile.org/Common/basic/Taxa.aspx?TaxonNameID=2516

Figs 29-33, 45

Coreus dentipes Fabricius 1803: 196.

Redescription. Holotype. Male. $\mathrm{n}=1$. Total body length: 9.8; head length: 1.1; head width: 1.6; eye width: 0.3 ; interocular space: 0.7 ; preocellar distance: 0.2 ; interocellar space: 0.3. Rostrum: (missing). Antennal segments length: I 2.6, II 2.4, III 1.4, IV 1.8. Pronotal length: 1.8; width: 2.8. Scutellar length: 1.3; width 1.1. Length of abdomen with hemelytra: 8.5 ; length of abdomen with hemelytra: 8.4 ; abdomen width: 2.4 . Dorsal coloration: Head light brown, except anterior region of ocelli and post ocular region dark brown, with many short hairs. Antennal segments 1-2 longer than 3-4, 4 longer than 3, segment 1-3 light brown, segment 4 dark brown except base, and segment 5 light brown. Pronotum are light brown except anterior margin dark brown. Scutellum brown except edges and posterior process dark brown. Connexival: two lateral segment light brown except lateral projection dark brown. Ventral coloration: light brown except area next to conexivum dark brown to brown, light brown not uniformy red. Legs: fore and middle femora dark brown, tarsi light brown with light brown spots. Mid femur with one spine distally. Fore femur dark brown with spines, basally very long, medially short and distally long. Structure: pronotum granulose; frontal angles granulose and projecting as acute spines reaching ocular tubercles; humeral angles with two rounded projections and shorter spines; scutellum granulose with pilosity. Hemelytra as long as the abdomen, brown with punctuations and pilosity, membrane dark brown.

Specimen examined. Argentina: Formosa: $10^{\lambda}$ Laguna Oca $\left(26^{\circ} 13^{\prime} 56.25^{\prime \prime}\right.$, $58^{\circ} 13^{\prime} 04.84^{\prime \prime W)}$.

Distribution. Argentina: Formosa: Laguna Oca.

Remarks. This is the first record of this species for Argentina

\section{Acknowledgments}

We want to thank our gratitude anonymous reviewers for the critical reading of the manuscript; to Dr M. Griffin (University of La Plata, Argentina) for revising the english. This study was supported by Consejo Nacional de Investigaciones Científicas y Técnicas de Argentina (CONICET).

\section{References}

Amyot CJB, Serville JGA (1843) Histoire naturelles des insectes. Hémiptères. In : «Suites à Buffon». Fain et Thunot, Paris, lxxvi + 681 pp., 12 pls. 
Bachmann AO (1999) Catálogo de los tipos de Heteroptera (Insecta) conservados en el Museo Argentino de Ciencias Naturales. Revista del Museo Argentino de Ciencias Naturales, Nueva Serie, 1: 191-230.

Berg C (1878) Hemiptera Argentina: Ensayo de una monografia de los Hemipteros Heteropteros y Homopteros de la República Argentina. Anales de la Sociedad Científica Argentina 6: 23-36, 82-89, 129-141, 179-192, 223-233, 261-284.

Berg C (1879) Hemiptera Argentina Enumeravit Specisque Novas, Bonariae, AR: Coni, Hamburgo, DE: Frederking et Graf, with continuous pagination,i-viii, 9-316.

Berg C (1892) Nova Hemiptera faunarum argentinae et uruguayiensis. Anales de la Sociedad Cientifica Argentina 33: 5-11, 43-50, 52-72, 82-96, 94-104, 151-165; 34: 193-205. [Reprint 1892, Pauli et Coni Filiorum Boniariae, 112 pp.]

Bergroth E (1912) Notes on Coreidae and Neididae. Annales de la Société Entomologique de Belgique 56: 76-92.

Blöte HC (1935) Catalogue of the Coreidae in the Rijksmuseum van Natuurlijke Historie. Part II. Coreinae, first part. Zoologische Mededeelingen 18: 181-227.

Bosq JM (1937) Lista preliminar de los Hemípteros (Heteropteros), especialmente relacionados con la agricultura nacional. Revista de la Sociedad Entomológica Argentina 9: 111-134.

Bosq JM (1940) Lista preliminar de los Hemípteros (Heteropteros), especialmente relacionados con la agricultura nacional (continuación). Revista de la Sociedad Entomológica Argentina 10: 399-417.

Brailovsky H (1987) Three new genera and six new species of Neotropical Coreidae (Heteroptera). Journal of the New York Entomological Society 95: 518-530.

Brailovsky H (1990) Revisión del complejo “Althos” (Hemiptera-Heteroptera-Coreidae). Publicaciones especiales Universidad Autónoma de México 5: 1-156.

Brailovsky H (1993) Género nuevo y especies nuevas de coreidos neotropicales (HemipteraHeteroptera-Coreidae: Acanthocerini, Chariesterini, Coreini, Discogastrini, Leptoscelidini, y Nematopodini) Anales Instituto Biología Universidad Nacional Autónoma México Serie Zoología 64: 109-127.

Brailovsky H, Barrera E (2003) Three new species and new distributional records of Discogastrini from Central and South America (Hemiptera: Heteroptera: Coreidae: Coreinae). Journal of the New York. Entomological Society 111 (1): 22-28. doi: 10.1664/0028-7199(2003)111[0022:TNSAND]2.0.CO;2

Burmeister HC (1835) Handbuch der Entomologie. Volume 2, 1835 1, i-xii + 1-400. T. Enslin, Berlin.

Casini CE (1989) Descripción de un nuevo género y especie de la tribu Acanthocerini (Hemiptera, Coreidae). Revista Brasileira de Entomologia 33: 25-29.

Coscarón MC (1998) Los ejemplares tipo de Coreoidea (Heteroptera) depositados en la colección del museo de La Plata. Serie Técnica y Didáctica, Museo de La Plata 34: 1-5.

Coscarón MC Coreidae. In: Catalogue of the Heteroptera or true bugs of Argentina. Zootaxa (In press)

Fabricius JC (1775) Systema Entomologiae, Sistens Insectorum Classes, Ordines, Genera, Species, Adjectis synonymis, locis, descriptionibus, observationibus. Flensburgi et Lipsiae, Korte. xxvii +832 pages. 
Fabricius JC (1803) Systema Rhyngotorum Secundum Ordines, Genera, Species, Adjectis Synonymis, Locis, Observationibus, descriptionibus. Reichard, Brunsvigae, i-x+335 pages.

Hayward JK (1942) Primera lista de insectos tucumanos perjudiciales Publicación Miscelanea, Estación Experimental Agrícola,Tucumán 1: 1-110.

Hayward JK (1960) Insectos Tucumanos perjudiciales. Revista Industrial y Agrícola de Tucumán 42:3-144. (1958).

Henry TJ. (2009) Biodiversity of Heteroptera, pp, 223-254 pp In: Foottit R, Adler P (Eds) Insect Biodiversity: Science and Society. Wiley- Blackwell, Oxford.

Kirkaldy GW (1904) Ueber Notonectiden (Hemiptera). II. Wiener Entomologische Zeitung 23: $111-135$.

Kormilev N (1954) Notas sobre Coreidae Neotropicales II; (Hemiptera) Mericorinae de la Argentina y países limítrofes. Revista Ecuatoriana de Entomología y Parasitología 2: 153-185. Lethierry L, Severin G (1894) Catalogue Général des Hémiptères. F. Hayez, Bruxelles and Berlin 1894: 1-277.

Mayr GL (1865) Diagnosen neuer Hemipteren II. Verhadlungen der Kaiserlich-königlichen Zoologisch-botanischen Geselleschaft in Wien 15: 429-446.

Mayr GL (1866) Hemiptera. Reise der Österreichischen Fregatte Novara um die Erde in den Jahren 1857, 1858, 1859 unter den Befehlen des Commodore B. von Wüllerstorf-Urbair. Novara Expedition. Zoologischer Theil. II. Abteilung 1. Karl Gerold's Sohn, Wien, 204 pp. Mitchell PL (2000). Leaf-Footed Bugs (Coreidae), pp, 337-403. In: Schaefer CW, Panizzi AR (Eds) Heteroptera of Economic Importance. CRC Pres, Boca Raton, USA.

O'Shea R (1973) Crinocerus Burmeister 1839 (Insecta: Hemiptera-Heteroptera: Coreidae): arequest for the validation of the commonly used type-species. Z.N.(S.) 2026. Bulletin of Zoological Nomenclature 30: 63-64.

O'Shea R (1980) A generic revision of the Acanthocerini (Hemiptera: Coreidae: Coreinae). Studies on Neotropical Fauna and Environment 15: 57-80. doi: 10.1080/01650528009360565

Packauskas RJ (1994) Key to the subfamilies and tribes of the New World Coreidae (Hemiptera), with a checklist of published keys to genera and species. Proceedings of the Entomological Society of Washington 96: 44-53.

Packauskas R (2010). Catalog of the Coreidae, or Leaf-Footed Bugs, of the New World. Fort Hays Studies, Fourth Series, Number 5, 270 pp.

Pennington MS (1920) Lista de los Hemipteros Heteropteros de la República Argentina. Primera parte. Pentamoidea- Coroidea, Buenos Aires 1-16.

Pennington MS (1921) Notas sobre Coreidos Argentinos. Physis, Buenos Aires 5: 28-39.

Quintanilla RH, Margheritis AE, Rizzo HF (1968) Catálogo de Hemípteros hallados en la provincia de Entre Ríos (Argentina). Revista de la Facultad de Agronomía y Veterinaria de Buenos Aires 47: 29-38.

Quintanilla RH, Margheritis AE, Rizzo HF (1976) Catálogo de hemípteros hallados en la provincia de Corrientes (Argentina). Revista de la Sociedad Entomológica Argentina 35: 115-133.

Quintanilla RH, Rizzo HF, Nuñez AS (1981) Catálogo preliminar de hemípteros hallados en la provincia de Misiones (Argentina). Revista de la Facultad de Agronomía, 2: 145-161. 
Rizzo HF (1976) Hemípteros de interés agrícola. Chinches perjudiciales y chinches benéficas para los cultivos. Editorial Hemisferio Sur, Buenos Aires, pp.69.

Schaefer CW (1964) The morphology and higher classification of the Coreoidea (HemipteraHeteroptera): Parts I and II. Annals of the Entomological Society of America 57: 670-684.

Schaefer CW (1965) The morphology and higher classification of the Coreoidea (HemipteraHeteroptera). Part III. The families Rhopalidae, Alydidae, and Coreidae. Miscellaneous Publications, Entomological Society of America 5: 1-76.

Schuh RT, Slater JA (1995) True Bugs of the World (Hemiptera: Heteroptera): Classification and Natural History. Cornell University Press, Ithaca and London. 366 pp.

Signoret V (1863) Revision des Hémiptères du Chile. Annales de la Société Entomologique de France Serie 4, 3: 541-588.

Stål C (1855) Nya Hemiptera. Öfversigt af Kongliga Vetenskaps-Akademiens Förhandlingar 12: $181-192$.

Stål C (1859) Till kännedomen om Coreida. Öfversigt af Kongliga Vetenskaps-Akademiens Förhandlingar 16: 449-475.

Stål C (1867) Bidrag till Hemipterernas systematik. Öfversigt af Kongliga Svenska Vetenskaps - Akademiens Förhandlingar 24: 491-560.

Stål C (1870) Enumeratio Hemipterorum: Bidrag till en företeckning öfver alla Hittils kanda Hemiptera, jemte systematiska meddelanden. Parts I-V. Kongliga Svenska Vetenskaps Akademiens Handlingar. Part I. 9: 1-232.

Viana MJ, Williner GJ (1972) Evaluación de la fauna entomológica y aracnológica de las provincias cuyanas. Primera comunicación. Acta Scientifica, Serie Entomología 5: 1-29.

Viana MJ, Williner GJ (1978) Evaluación de la fauna entomológica y aracnológica de las provincias centrales y cuyanas (cuarta comunicación). Acta Scientifica, Serie Entomología 11: 1-77. 